\title{
INTEGRABILITY OF FOURIER TRANSFORMS UNDER AN ERGODIC HYPOTHESIS
}

\author{
GAVIN BROWN \\ (Received 12 July 1977) \\ Communicated by A. P. Robertson
}

\begin{abstract}
The object is to unify and complement some recent theorems of Hewitt and Ritter on the integrability of Fourier transforms, but the underlying theme is the ancient one that Plancherel's theorem is the "only" integrability constraint on Fourier transforms. The distinguishing feature of the results is that we restrict attention to positive measures (or functions) which satisfy an ergodic condition and whose transforms are positive. (In fact we employ sums of discrete random variables, a technique which seems to have been largely ignored in context.) The general setting is that of locally compact abelian groups but we are chiefly interested in the line or the circle, and it appears that the theorems are new for these classical groups.
\end{abstract}

Subject classification (Amer. Math. Soc. (MOS) 1970): primary 42 A 68, 42 A 73, 43 A 25.

Keywords: Fourier-Stieltjes transforms, discrete random variables.

\section{Introduction}

(1.1) Scheme. The general background is discussed in the next sub-section, the notation to be used is listed in (1.3) and the main theorem stated and discussed in (1.4) and (1.5). Some basic technical preliminaries are gathered in Section 2. The business of Section 3 is the proof of the main result for the real line (and circle). The extension to locally compact abelian groups is proved in Section 4. In particular it should be possible (and desirable) for the reader to pass directly from Section 1 to Sections 3 and 4 using Section 2 only for occasional reference. Formulae are numbered consecutively, with a fresh start in each of the four sections.

(1.2) Background and general description. A fundamental question of harmonic analysis concerns the relationship between species of "good" behaviour of a function or measure and the convergence properties of its Fourier transforms. There are various positive results in which smoothness of a function implies

(C) Copyright Australian Mathematical Society 1978

Copyright. Apart from any fair dealing for scholarly purposes as permitted under the Copyright Act, no part of this JOURNAL may be reproduced by any process without written permission from the Treasurer of the Australian Mathematical Society. 
smallness of its transform, but we are concerned here with interpreting the question: "How does integrability of a measure condition integrability of its transform?" In this we are following two recent papers by Hewitt and Ritter $(1976,1977)$.

A partial answer to the question just posed has been well known for over fifty years-in general, the only constraints are imposed by the theorems of RieszFischer, Plancherel and Hausdorff-Young. (Curiously though, explicit theorems demonstrating even this "answer in principle" for the case of the real line do not appear to have been given prior to the Hewitt-Ritter work just cited.) A convenient focus is a theorem proved by Gronwall (1921), or see Zygmund (1959).

If $\varphi:] 0, \infty\left[\rightarrow\left[0, \infty\left[\right.\right.\right.$ satisfies $\lim _{s \rightarrow 0} \varphi(s)=\infty$, then there is a continuous function $f$ on the circle such that

$$
\sum_{n=-\infty}^{\infty}\left|f^{\wedge}(n)\right|^{2}<\infty, \quad \sum_{n=-\infty}^{\infty}\left|f^{\wedge}(n)\right|^{2} \varphi\left(\left|f^{\wedge}(n)\right|^{-1}\right)=\infty .
$$

This rules out pure integrability-to-integrability results from $L_{p}(\mathbf{T})$ to $l_{\infty}(\mathbf{Z})$, and does so, in a strong way, because of the continuity condition imposed.

In the opposite direction there is a corpus of theorems which obtain integrability properties of $f^{\wedge}$ from integrability properties of $f$, provided that $f$ satisfies some additional constraints. (A survey of a particular class of these is given by Boas (1967). The side-conditions typically involve positivity of $f$ or of $f^{\wedge}$.)

Accordingly, by a "theorem of Gronwall type" we mean a result which establishes sharp failure of integrability-to-integrability statements for some class of well-behaved measures (functions).

All the main results of Hewitt and Ritter $(1976,1977)$ are theorems of Gronwall type in this sense. In particular, all measures considered in Hewitt and Ritter (1977) are positive and have positive transforms. (As we noted above, this can be classed as significantly good behaviour in the context of the theorems exposed in Boas (1967).) On the other hand, for the absolutely continuous functions discussed in Hewitt and Ritter (1976), the side-condition is continuity in the presence of compact support-in other words, the original Gronwall condition. (We have discussed an alternative route to this kind of result in Brown (1977b).)

However, one does not obtain a theorem of Gronwall type where both kinds of constraint are imposed, because there is the (easy) integrability-to-integrability result that, if $f \in L_{1}(\mathbf{R}), f$ is continuous at 0 , and $f^{\wedge} \geqslant 0$, then $f^{\wedge} \in L_{\mathbf{1}}(\mathbf{R})$. It should also be noted that the apparent weakening of theorems of Gronwall type which arises when the functions are required only to be bounded as opposed to continuous is largely illusory because one may take a convolution product with a suitable function in $L_{1}$. Such a method was used by Salem and Zygmund (1947) and the analogue on the real line is what underpins Brown (1977b).

Thus we may say that Brown (1977b) and Hewitt and Ritter (1976) are concerned with theorems of Gronwall type in the absolutely continuous case with boundedness constraint, while Hewitt and Ritter (1977) consider theorems of Gronwall type 
in the singular case with positivity constraint. In addition, many different proof techniques are employed in Hewitt and Ritter $(1976,1977)$ (general Riesz products, $\Lambda_{2}$-sets, Rudin-Shapiro polynomials, lifting from $\mathbf{T}$ to $\mathbf{R}$, systematic translation and dilation) and, in general, objects constructed are highly irregular under translation.

In our main theorem we impose the constraint of a regular pattern of construction (the convenient formalization being ergodicity with respect to a countable subgroup) on both functions and measures, and demand also that all transforms, functions and measures be positive. In this way we obtain an omnibus theorem of Gronwall type whose method of proof is uniform throughout all cases, which sharpens the results of Hewitt and Ritter (1977) and gives a unified treatment complementary to the various results of Hewitt and Ritter (1976). Moreover, the real line is as natural as the circle group from the standpoint of our methods. We must pay for all this, in the absolutely continuous case, by removing the boundedness constraint on the functions involved. Indeed our functions are generically unbounded and, in view of our insistence on translation regularity, this means unbounded in every neighbourhood which intersects the support. Because boundedness was used to force the integrability properties in the original Gronwall theorem, we must include some such constraints explicitly. In fact it is possible to make the best possible demand and insist that the functions belong to $L_{p}$, for every positive $p$.

I am grateful to the authors of Hewitt and Ritter $(1976,1977)$ for prepublication copies of their work, and take pleasure in acknowledging some stimulating conversations with Edwin Hewitt on the integrability of Fourier transforms.

(1.3) Notation. In general the symbol $G$ denotes a non-discrete locally compact abelian group, and $X$ the character group of $G$. Haar measure on $G$ is denoted by $\lambda$ and on $X$ by $\theta$.

In the cases of special interest, namely when $G$ is the real line, $\mathbf{R}$, or the circle group, $\mathbf{T}$, we choose specific realizations as follows: For $\boldsymbol{G}=\mathbf{R}$ we take $\lambda$ as Lebesgue measure and identify $X$ with $\mathbf{R}$ according to the correspondence $x \leftrightarrow \chi$, where

$$
\chi(y)=\exp (2 \pi i x y) \quad(y \in \mathbf{R}) .
$$

(In fact we also find it convenient to employ the notation $e(-)$ as an alternative for $\exp (2 \pi i-)$.) It is, of course, natural to take $\theta$ to be $(1 / 2 \pi) \lambda$. The circle group is realized as the closed interval $\left[-\frac{1}{2}, \frac{1}{2}\right]$ with the end points identified and with addition defined modulo one. With this realization of $G=\mathbf{T}$, we take $\lambda$ as Lebesgue measure on ] $-\frac{1}{2}, \frac{1}{2}$ ] and identify $X$ with the additive group $Z$ of integers. Here the character $\chi$ represented by the integer $n$ is given by

$$
\chi(t)=e(n t) \quad(t \in \mathbf{T})
$$

and $\theta$ is taken to be counting measure. 
There will be occasion to consider also the cyclic group, $\mathbf{Z}(a)$, of order $a$, and the group, $\Delta_{q}$, of $q$-adic integers, but the details can be left until needed in Section 4.

The Fourier-Stieltjes transform of a measure $\mu$ on $G$ is the function $\mu^{\wedge}$ defined by

$$
\mu^{\wedge}(\chi)=\int \bar{\chi}(g) d \mu(g) \quad(\chi \in X) .
$$

For $p>0, L_{p}(G)$ denotes the space of (equivalence classes modulo $\lambda$ ) of $p$-integrable functions, and $L_{p}(X)$ has a similar significance. For $f \in L_{1}(G)$, the Fourier transform $f^{\wedge}$ of $f$ coincides with the Fourier-Stieltjes transform of the measure $f$ whose Radon-Nikodym derivative with respect to $\lambda$ is $f$.

$C_{0}(X)$ (respectively $C_{0}^{+}(X)$ ) denotes the continuous (respectively non-negative continuous) functions on $X$ which vanish at infinity. By a probability measure on $G$ we shall mean a positive regular bounded Borel measure on $G$ which has total mass one. For such a measure $\mu$ the involute $\tilde{\mu}$ is the unique probability measure characterized by the formula

$$
\left(\tilde{\mu}^{\wedge}\right)(\chi)=\mu^{\wedge}(\chi)^{-} \quad(\chi \in X) .
$$

$\mu$ is called hermitian if $\mu=\tilde{\mu}$. Note that if $\mu$ is any probability measure on $G$ whose Fourier-Stieltjes transform vanishes at infinity then the convolution product $\mu * \tilde{\mu}$ is a hermitian probability measure on $G$ whose Fourier-Stieltjes transform belongs to $C_{0}^{+}(X)$.

Let $D$ be a countable subgroup of $G$. A Borel set $E$ is $D$-invariant if the set $\{x+d: x \in E\}$ is contained in $E$ for every $d$ in $D$. A probability measure $\mu$ on $G$ is $D$-ergodic if $\mu$ assigns to each $D$-invariant Borel set either mass one or mass zero (that is, $\mu$ is concentrated on every non-negligible $D$-invariant Borel set). An example of this phenomenon is a uniform mass distribution over the classical Cantor set in $\mathbf{R}$. The resulting measure is $D$-ergodic when $D$ is chosen as the group of triadic rationals. We shall say that a probability measure $\mu$ on $G$ is ergodic if there exists some countable subgroup $D$ with respect to which $\mu$ is $D$-ergodic, and, to avoid difficulties in the non-metrizable case, we include also measures of the form $\lambda_{K} * \mu$, where $K$ is a compact subgroup of $G$ and the image of $\mu$ in $M(G / K)$ (under the canonical map $\pi_{K}^{*}$ induced by the quotient map) is $D$-ergodic. The class $\mathscr{E}$ of ergodic measures is then stable under quotients, in the sense that $\mu \in M(G), \mu \in \mathscr{E}$ implies $\pi_{K}^{*}(\mu) \in \mathscr{E}$, for any compact subgroup $K$ of $G$. (See Proposition 5 in (4.4) below.)

\section{(1.4) Main result.}

THEOREM. Let $G$ be a nondiscrete locally compact abelian group. Suppose that $1 \leqslant p<\infty$ and that $\varphi:] 0, \infty[\rightarrow] 0, \infty[$ is a measurable function such that $\varphi(x) \rightarrow \infty$ as $x \rightarrow 0$. Then there exist measures $\sigma_{p}, \tau_{y}$ on $G$ whose Fourier-Stieltjes transforms 
belong to $C_{0}^{+}(X)$ and satisfy

(i) $\int \sigma_{p}(\chi)^{p} d \theta(\chi)<\infty, \quad \int \hat{\sigma_{p}}(\chi)^{p} \varphi\left(\hat{\sigma_{p}}(\chi)\right) d \theta(\chi)=\infty$,

(ii) $\int \tau_{p}^{\wedge}(\chi)^{p} d \theta(\chi)=\infty, \int \tau_{p}(\chi)^{p}\left(\varphi\left(\tau_{p}^{\wedge}(\chi)\right)\right)^{-1} d \theta(\chi)<\infty$.

Moreover $\sigma_{p}, \tau_{p}$ may be chosen as ergodic probability measures with support $a$ compact neighbourhood of zero, and the following additional properties:

The measures $\sigma_{p}$, for $p>2$, and $\tau_{p}$, for $p \geqslant 2$, are singular to Haar measure $\lambda$ on $G$. In the remaining cases $\sigma_{p}, \tau_{p}$ are absolutely continuous, and their Radon-Nikodym derivatives with respect to $\lambda$ belong to $\bigcap_{r>0} L_{r}(G)$.

Corollary. For $1 \leqslant p<\infty$, an ergodic probability measure $\sigma$, whose transform is nonnegative and vanishes at infinity, may be chosen to satisfy either

$$
\boldsymbol{\sigma}^{\wedge} \text { belongs to } L_{r}(X) \text { if and only if } r \geqslant p,
$$

or

$$
\boldsymbol{\sigma}^{\wedge} \text { belongs to } L_{r}(X) \text { if and only if } r>p \text {. }
$$

Proof of Corollary. To obtain (i) take $\varphi(x)=\log \left(x^{-1}\right)$ and $\sigma=\sigma_{p}$ in the theorem. To obtain (ii) take $\varphi(x)=\log \left(x^{-1}\right)$ and $\sigma=\tau_{p}$ in the theorem.

(1.5) Comments. The main interest is, of course, when $\varphi$ tends very slowly to infinity as $x \rightarrow 0$. There is no loss of generality in assuming that $\varphi$ is decreasing in the wide sense and we can allow $\sigma_{p}, \tau_{p}$ to take the value zero by making the obvious interpretation of the values of the integrands at the appropriate points. (In fact on the classical groups we construct measures with strictly positive transforms.)

We are now in a position to explain in technical terms how the main theorem fits into the complex of existing work. It is natural to concentrate mainly on the relationship with the recent papers (Hewitt and Ritter, 1976, 1977), bearing in mind the historical comments given there.

The closest point of contact with the Hewitt-Ritter work concerns the singular measures $\tau_{p}$, for $p \geqslant 2$, which appear in the statement. Indeed the special case which asserts the existence of $\tau_{2}$ is effectively Theorem 9.2 of Hewitt and Ritter (1977), sharpened by the addition of the ergodic property. By considering also $2<p<\infty$, we are able to incorporate also (a) of Theorem 2.2 of Hewitt and Ritter (1977). In this connexion recall that the original Gronwall theorem demonstrates a species of left-handed discontinuity in the sense that it considers transforms which fail to belong to $L_{r}(Z)$ for $r<2$ but belong to $L_{r}(Z)$ for $r \geqslant 2$. (It would be more accurate to associate this discontinuity phenomenon, per se, with the name of 
Carleman, since it is the sharpening which arises from consideration of the function $\varphi$ that corresponds to Gronwall's contribution.) Theorem 9.2 of Hewitt and Ritter (1977) (even specialized to the circle) gives not only the first Gronwall analogue for singular measures, but also the first right-handed discontinuity result in the sharp Gronwall form-the underlying pattern involves transforms which fail to belong to $L_{r}(X)$ for $r \leqslant 2$ but belong to $L_{r}(X)$ for $r>2$. In the terminology just coined, our earlier remark about Theorem 2.2(a) of Hewitt and Ritter (1977) becomes the comment that consideration of $\tau_{p}$ (rather than only $\tau_{2}$ ) allows us to treat a left-handed discontinuity at any $p$ in the range $\left[2, \infty\left[\right.\right.$. By considering also $\sigma_{p}$, for $p>2$, we are able to find a Gronwall analogue for singular measures which establishes a right-handed discontinuity at any $p$ in the range $] 2, \infty[$. Thus we can absorb also (b) of Theorem 2.2 of Hewitt and Ritter (1977) into our version of a Gronwall theorem for singular measures.

Still concentrating on the relationship between the singular case of our theorem and the main results of Hewitt and Ritter (1977), we note that our theorem is formally stronger, because we insist on ergodic measures, and that our methods are different, because we use infinite convolutions of discrete measures. Hewitt and Ritter employed general Riesz products.

The first point to be noted is that ergodicity can also be achieved with Riesz products although these measures are not typically ergodic. (In fact it was shown in Brown (1975) that a Riesz product is ergodic if the dissociate set used in its construction satisfies certain (strong) number-theoretic constraints, and it was noted that a Riesz product may fail to be ergodic for every countable subgroup.) It follows that, at least in the case of the circle, the singular case of our theorem could be proved along the lines used in Hewitt and Ritter (1977) and, presumably, a development of the ideas of (7.2) of Brown (1975) in the light of the structure theory of discrete abelian groups would make available such a proof for general compact abelian groups.

Our main reason for choosing to work with infinite convolutions springs from quite separate considerations. The advantage is that we obtain a simple and natural construction on the real line-indeed there is no essential difference between our treatment of the line and the circle. In contrast the Riesz product approach used in Hewitt and Ritter (1977) requires that $\mathbf{R}$ be approached indirectly by "lifting" from its quotient $\mathbf{R} / \mathbf{Z}$. That particular lifting process, although well understood, can be rather troublesome. This is unlike passage from a quotient of the form $G / K$, where $K$ is compact, a process which lends itself to general arguments (cf. Ritter $(1968,8.2 .7)$, and Brown $(1973,2.5))$. Thus one has both practical and aesthetic motivation for giving a direct construction on the line.

Given that we use infinite convolutions on $\mathbf{R}$ and $\mathbf{T}$, it is natural that we should use a similar method for general groups. However, it should be noted that, away from the case $G=\mathbf{R}$, Riesz products offer certain advantages. Once one has gone 
through the structure theoretical arguments inherent in the choice of a dissociate set, the question of singularity or absolute continuity of the resulting Riesz product can be settled without further reference to the particular choice of compact abelian groups. This follows from a theorem of Brown and Moran (1974), and avoids consideration of special groups. In fact, if ergodicity and some other technical properties are set aside, the neatest approach seems to be to use infinite convolutions on $\mathbf{R}$ (and $\mathbf{T}$ ) and Riesz products on compact abelian groups (including $\mathbf{T}$ )! However we go about things, it is necessary to consider some, if not all, of the stability properties appearing in the statement of Proposition 4 (of Section 2 below) in order to obtain results valid for all nondiscrete locally compact abelian groups.

The remaining cases of our theorem complement the results of Hewitt and Ritter (1976) but do not contain them. What we show here is that the same methods which apply to singular measures can be extrapolated to absolutely continuous measures. There they give theorems of Gronwall type which establish left-handed and right-handed discontinuities when $p$ does not exceed 2. All the usual examples in the absolutely continuous case focus on continuity of the Radon-Nikodym derivative and are either violently non-ergodic or involve rapid change of argument. The question arises, however, whether the methods in Hewitt and Ritter (1977) could also be extrapolated to the case of absolutely continuous measures. Here a problem arises because we want the measure, say $\mu$, under consideration to have a compact neighbourhood for its support and also the property that $d \mu / d \lambda \in \bigcap_{p>0} L_{p}(G)$. For the Riesz products $\mu$ which would arise, these properties were established in Hewitt and Zuckerman (1967) for $\mu * \mu$ (rather than for $\mu$ itself). The difficulty concerning the support of $\mu$ was resolved in Brown (1975), but there appears to be an essential problem concerning the integrability of $(d u / d \lambda)^{p}$, for large $p$. We have no such difficulties with infinite convolutions. Moreover, ergodicity is guaranteed by the results in Brown and Moran (1973).

\section{Technical preliminaries}

(2.1) Summability of sequences. We require some elementary estimates concerning summability of sequences which arise in connexion with the function $\varphi$ which appears in the statement of Gronwall's theorem. The proof of part (i) of Proposition 1 can be compared with the proof of Theorem 7.5 of Chapter XII of Zygmund (1959). The sharpening which arises from consideration of an auxiliary sequence such as $\left(a_{n}\right)$ was used in a related context (for $p=2$ ) in Brown (1977b). Assertion (ii) is a variant of Lemma 9.5 of Hewitt and Ritter (1977) but we offer a simpler argument.

Proposition 1. Suppose that $1 \leqslant p<\infty$ and that $\varphi:] 0, \infty[\rightarrow] 0, \infty[$ is a function such that $\lim _{g \rightarrow 0} \varphi(s)=\infty$. Suppose further that $\left(a_{n}\right)$ is an arbitrary pth power 
summable sequence of numbers in $[0,1]$ and that $C$ is a positive constant greater than one. Then there exist sequences $\left(s_{n}\right),\left(t_{n}\right)$, with $0<s_{n}, t_{n} \leqslant 1$, such that

(i) $\sum_{n=1}^{\infty} s_{n}^{p} \varphi\left(2 s_{n}\right)=\infty, \sum_{n=1}^{\infty} s_{n}^{p}<\infty, \quad s_{n} \geqslant a_{n}, \quad n=1,2, \ldots$,

(ii) $\sum_{n=1}^{\infty}\left(t_{n}^{p} / \varphi\left(2 t_{n}\right)\right) \prod_{j=1}^{n-1}\left(1+C t_{j}^{p}\right)<\infty, \quad \sum_{n=1}^{\infty} t_{n}^{p}=\infty$.

Moreover, it may be stipulated that

$$
\begin{aligned}
& \sum_{n=1}^{\infty} s_{n}^{r}=\infty, \text { whenever } 1 \leqslant r<p, \\
& \sum_{n=1}^{\infty} t_{n}^{r}<\infty, \quad \text { whenever } r>p .
\end{aligned}
$$

Proof. Let us define, for all $x>0$,

$$
\Phi(x)=\inf \{\min (\varphi(t), \log (1 / t)): 0<t \leqslant x\} .
$$

Observe that $\Phi:] 0, \infty[\rightarrow] 0, \infty[$ is decreasing in the wide sense and satisfies $\lim _{x \rightarrow 0} \Phi(x)=\infty$. In addition, the truth of (i) and (ii) when $\varphi$ is replaced by $\Phi$ implies not only these assertions for $\varphi$ but also the final summability assertions concerning $s_{n}^{r}, r>p$, and $t_{n}^{r}, 1 \leqslant r<p$. Accordingly, it will suffice simply to prove that (i), (ii) can be attained in the special case where $\varphi$ is nonincreasing. Let us make that assumption and proceed to give inductive definitions of $\left(s_{n}\right)$ and $\left(t_{n}\right)$. In the interests of clarity we keep these definitions completely distinct.

Definition of $\left(s_{n}\right)$ : Choose a positive decreasing sequence $\left(b_{n}\right)$ such that $0<b_{n} \leqslant 1$, $n=1,2, \ldots$, and

$$
\varphi(2 x)>n \text { whenever } 0<x \leqslant b_{n} .
$$

At the $j$ th stage of an induction choose positive integers $p(j), q(j)$, and a positive real number $x(j)$ such that

and

$$
\begin{gathered}
p(j)>p(j-1)+q(j-1), \\
\sup \left\{a_{n}: n \geqslant p(j)\right\} \leqslant x(j) \leqslant b_{j},
\end{gathered}
$$

$$
q(j) x(j)^{p}=j^{-2} \text {. }
$$

(Because $\Sigma a_{n}^{p}<\infty$, there is no difficulty in choosing $p(j)$ large enough to ensure that the supremum appearing in (3) is less than, say, $\frac{1}{2} b_{j}$. It is then a simple matter to make the required choice consistent with (2), (3) and (4).)

Now define, for positive integers $n$,

$$
\begin{aligned}
s_{n}=x(j), & \text { whenever } p(j) \leqslant n \leqslant p(j)+q(j)-1, \text { for some } j=1,2, \ldots, \\
s_{n}=a_{n}, & \text { for all other values of } n .
\end{aligned}
$$


The only case in which $s_{n}$ differs from $a_{n}$ is when $s_{n}=x(j)$, with $p(j) \leqslant n$. It is clear therefore that the left-hand side of (3) ensures that $s_{n} \geqslant a_{n}$, for all positive integers $n$. In addition, we see, by ignoring some indices and applying (4), (3) and (1), that

$$
\sum_{n=1}^{\infty} \varphi\left(2 s_{n}\right) s_{n}^{p} \geqslant \sum_{j=1}^{\infty} q(j) x(j)^{p} \varphi(2 x(j)) \geqslant \sum_{j=1}^{\infty} j^{-2} j=\infty .
$$

On the other hand we can estimate the two cases in the definition of $\left(s_{n}\right)$ separately (using (4) in conjunction with the second) to see that

$$
\left.\sum_{n=1}^{\infty} s_{n}^{p} \leqslant \sum_{n=1}^{\infty} a_{n}^{p}+\sum_{j=1}^{\infty} q(j) x j\right)^{p}<\infty .
$$

Definition of $\left(t_{n}\right)$ : For $0<y<C^{-1}$, let $N(y)$ be the least positive integer such that

$$
2 \leqslant\left(1+C y^{p}\right)^{N(y)} \leqslant 3 .
$$

Choose a positive decreasing sequence $(y(j))$ in $] 0, C^{-1}$, such that

$$
\varphi(2 y(j))>10^{j}, j=1,2, \ldots, \text { and } N(y(j)) \text { is strictly increasing. }
$$

Now define, for positive integers $n$,

$$
\begin{array}{ll}
t_{n}=y(1), & 1 \leqslant n \leqslant N(y(1)), \\
t_{n}=y(j), & \sum_{i=1}^{j-1} N(y(i))<n \leqslant \sum_{i=1}^{j} N(y(i)), \quad j=2,3, \ldots .
\end{array}
$$

(In view of the last part of (6) this defines $t_{n}$ for all positive integers $n$.) It follows from this definition of $\left(t_{n}\right)$ that

$$
\begin{aligned}
& \sum_{n=1}^{\infty}\left(t_{n}^{p} / \varphi\left(2 t_{n}\right)\right) \prod_{j=1}^{n-1}\left(1+C t_{j}^{p}\right) \\
& \quad \leqslant \sum_{j=1}^{\infty} \varphi(2 y(j))^{-1} N(y(j)) y(j)^{p} \prod_{i=1}^{j}\left(1+C y(i)^{p}\right)^{N(y(i))} \\
& \leqslant \sum_{j=1}^{\infty} \varphi(2 y(j))^{-1} \prod_{i=1}^{j}\left(1+C y(i)^{p}\right)^{2 N(y(i))} \\
& \quad \leqslant \sum_{j=1}^{\infty} 3^{2 j} \varphi(2 y(j))^{-1},
\end{aligned}
$$

where the final step follows from (5). Of course (6) demonstrates finiteness of the last sum. It remains only to note, from (5), that

$$
\begin{aligned}
\prod_{n=1}^{\infty}\left(1+C t_{n}^{p}\right) & \geqslant \prod_{j=1}^{\infty}\left(1+C y(j)^{p}\right)^{N(y(j))} \\
& \geqslant 2^{k}, \quad k=1,2, \ldots .
\end{aligned}
$$

It follows that $\Sigma t_{n}^{p}$ diverges and the proof of the proposition is complete. 
(2.2) Singularity of measures. We need a criterion for singularity of measures of the type which arise as distributions from coin-tossing experiments. The result quoted next is taken from Brown (1977a) and is a simplification of the basic technical lemmas of Brown and Moran (1975b).

Proposition 2. Let $P_{1}, P_{2}$ be probability measures on the measurable space $(\Omega, \mathscr{E})$ and let $E_{i}$ denote expectation with respect to $P_{i}$, for $i=1,2$. Suppose that there exists a sequence $\left(X_{n}\right)$ of complex random variables on $(\Omega, \mathscr{E})$ (square integrable with respect to $\left.P_{1}, P_{2}\right)$ such that

(i) $\sum_{k=0}^{\infty} \sup _{i, n}\left|E_{i}\left(X_{n} \bar{X}_{n+k}\right)-E_{i}\left(X_{n}\right) E_{i}\left(\bar{X}_{n+k}\right)\right|<\infty$,

(ii) $\sum_{n=1}^{\infty}\left|E_{1}\left(X_{n}\right)-E_{2}\left(X_{n}\right)\right|^{2}=\infty$

Then $P_{1}, P_{2}$ are mutually singular.

(2.3) The independent case. There would be no need for Proposition 2 if we could work exclusively with products of independent random variables; in which case all questions would be answered by the next proposition. The result in question is essentially Kakutani's criterion for orthogonality of product measures, which we quote in the form stated and proved in Brown and Moran (1975b). Because lack of independence militates against singularity rather than the other way about, this proposition serves as a general criterion for absolute continuity. It will be particularly useful in Section 4 where we consider products of cyclic groups because there one does have independence.

Proposition 3. Let $\left(X_{n}\right)$ be a sequence of independent complex random variables such that $\Pi E\left(\left|X_{n}\right|^{\top}\right)$ converges for some $r>1$. Then $\Pi X_{n}$ converges almost surely and in $L_{r}$ if and only if $\Pi E\left(X_{n}\right)$ converges.

(2.4) General groups. Our final technical preliminary concerns extension to general locally compact abelian groups. It handles results which are known in the following special cases: $\mathbf{R} ; \mathbf{T} ; \Pi_{n=1}^{\infty} \mathbf{Z}\left(m_{n}\right)$, where the $m_{n}$ are arbitrary integers not less than 2; $\Delta_{q}$, where $q$ is prime. The proposition is quoted from Brown (1973) but was extracted from the work in Stromberg (1968). All groups appearing in the statement are assumed locally compact and abelian.

Proposirion 4. Let $\mathscr{G}$ be the smallest class of locally compact abelian groups which contains the special cases enumerated above and which has the stability properties:

(i) If $G=H_{1} \times H_{2}$, where $H_{1} \in \mathscr{G}$ then $G \in \mathscr{G}$. 
(ii) If $K$ is a compact subgroup of $G$ and $G / K \in \mathscr{G}$ then $G \in \mathscr{G}$.

(iii) If $H$ is an open subgroup of $G$ and $H \in \mathscr{G}$ then $G \in \mathscr{G}$.

Then $\mathscr{G}$ is the class of all non-discrete locally compact abelian groups.

\section{The real line and the circle}

The proof will be broken down into a sequence of lemmas. Throughout we employ measures whose Fourier-Stieltjes transforms are of the form

$$
\mu^{\wedge}(x)=\prod_{n=1}^{\infty}\left(\cos ^{2}\left(2^{-n-1} x\right)+r_{n} \sin ^{2}\left(2^{-n-1} x\right)\right),
$$

where $\left(r_{n}\right)$ is a sequence of positive real numbers not greater than one. Any such measure may be exhibited as an infinite convolution,

$$
\mu=\underset{n=1}{*} \mu_{n}
$$

of discrete probability measures, defined by

$$
\mu_{n}=\frac{1}{2}\left(1+r_{n}\right) \delta(0)+1\left(1-r_{n}\right)\left(\delta\left(2^{-n}\right)+\delta\left(-2^{-n}\right)\right),
$$

where $\delta(a)$ denotes the probability measure concentrated at $a$. It is clear from (2) and (3) that $\mu$ is a probability measure which is ergodic with respect to the group of dyadic rationals and obvious from (1) that $\mu^{\wedge}$ is nonnegative. This can also be verified in a more complicated way if we note that $\mu_{n}=\left(1-r_{n}\right)\left(\rho_{n} * \tilde{\rho}_{n}\right)+r_{n} \delta(0)$, where $\rho_{n}=\frac{1}{2} \delta\left(2^{-n-1}\right)+\frac{1}{2} \delta\left(-2^{-n-1}\right)$. This point will recur in (4.1), (4.2), where it no longer appears artificial. A simple proof of the fact that the Fourier-Stieltjes transform of $\mu$ vanishes at infinity if and only if $r_{n} \rightarrow 0$ can be found in Brown (1973, p. 503).

So far it has made no difference whether we regarded the measure $\mu$ as defined on the line or on the circle. For the sake of definiteness we will now fix attention on the line. Some mild modifications (simplifications in the main) give the corresponding results for the circle.

Unlike Riesz products, measures such as $\mu$ do not offer exact interpolation by their transforms. In our present context this is not a serious drawback as the first lemma shows.

LeMmA 1. There is a positive constant A (independent of the choice of $\left(r_{n}\right)$ ) such that, for $-\pi \leqslant x \leqslant \pi$,

$$
r_{n}+4^{1-n} \geqslant \mu^{\wedge}\left(x+2^{n} \pi\right) \geqslant A r_{n}, \quad n=1,2, \ldots .
$$


Proof. Because each term in the product (1) is not greater than one, we may obtain an upper bound by estimating a single term. In fact

$$
\begin{aligned}
\mu^{\wedge}\left(x+2^{n} \pi\right) & \leqslant \cos ^{2}\left(2^{-n-1} x+\frac{1}{2} \pi\right)+r_{n} \sin ^{2}\left(2^{-n-1} x+\frac{1}{2} \pi\right) \\
& \leqslant \sin ^{2}\left(2^{-n-1} \pi\right)+r_{n},
\end{aligned}
$$

and this gives the required upper bound.

In the other direction, note that

$$
\begin{aligned}
\mu_{n}^{\hat{n}}\left(x+2^{n} \pi\right) & =\cos ^{2}\left(\frac{1}{2} \pi+2^{-n-1} x\right)+r_{n} \sin ^{2}\left(\frac{1}{2} \pi+2^{-n-1} x\right) \\
& \geqslant r_{n} \cos ^{2}(\pi / 4) .
\end{aligned}
$$

Moreover, if $k$ is a positive integer strictly less than $n$,

$$
\mu_{\hat{k}}\left(x+2^{n} \pi\right)=\mu_{\hat{k}}(x) \geqslant \cos ^{2}\left(2^{-k-1} \pi\right) ;
$$

while, for $k=n+1, n+2, \ldots$,

$$
\begin{aligned}
\mu_{k}\left(x+2^{n} \pi\right) & \geqslant \cos ^{2}\left(2^{n-k-1} \pi \pm 2^{-k-1} \pi\right) \\
& \geqslant \cos ^{2}\left(\pi\left(2^{n}+1\right) / 2^{k+1}\right) .
\end{aligned}
$$

The proof of (4) is now an easy consequence of the remark that the infinite product, $\Pi \cos ^{4}\left(2^{-k-1} \pi\right)$, converges.

The estimates in the previous lemma apply only to a subset of values of the transform. Now we obtain some extra information. Let us write

$$
P_{n}(x)=\prod_{j=1}^{n}\left(\cos ^{2}\left(2^{-j-1} x\right)+r_{j} \sin ^{2}\left(2^{-j-1} x\right)\right)=\prod_{j=1}^{n} \hat{\mu_{j}}(x),
$$

and

$$
W_{n}=\left\{\sum_{r=0}^{n} \varepsilon_{r} 2^{r} \pi: \varepsilon_{r}=0,1\right\} .
$$

LEMMA 2. For any $x \in[-\pi, \pi], p \in[1, \infty[, n=1,2, \ldots$,

$$
\sum_{w \in W_{n}} P_{n}(x+w)^{p} \leqslant \prod_{j=1}^{n}\left(1+r_{j}^{p}\right) .
$$

Proof. Because $P_{n-1}(x+w)=P_{n-1}\left(x+w+2^{n} \pi\right)$, we see that the term on the left-hand side of (7) equals

$$
\sum_{w \in W_{n-1}} P_{n-1}(x+w)^{p}\left(\mu_{n}^{\hat{n}}\left(x+w+2^{n} \pi\right)^{p}+\mu_{n}(x+w)^{p}\right) .
$$

However, writing $\theta_{n}=2^{-n-1}(x+w)$, we have

$$
\begin{aligned}
\mu_{n}(x+w) & =\cos ^{2} \theta_{n}+r_{n} \sin ^{2} \theta_{n}, \\
\mu_{n}^{\hat{n}}\left(x+w+2^{n} \pi\right) & =\sin ^{2} \theta_{n}+r_{n} \cos ^{2} \theta_{n} .
\end{aligned}
$$

It follows, from the convexity of the map $t \rightarrow t^{p}$, that

and the rest is clear.

$$
\mu_{n}(x+w)^{p}+\mu_{n}\left(x+w+2^{n} \pi\right)^{p} \leqslant 1+r_{n}^{p},
$$


Lemma 2 will be used in direct fashion for an integral estimate, but we also use it in obtaining a more elaborate result of the same type.

LEMMA 3. Suppose that $p \geqslant 1$ and that $\psi$ is a nonnegative nondecreasing measurable function which satisfies

$$
\sum_{n=1}^{\infty} \psi\left(2 r_{n}\right) r_{n}^{p} \sum_{j=1}^{n-1}\left(1+r_{j}^{p}\right)<\infty
$$

Then

$$
\int_{-\infty}^{\infty} \mu^{\wedge}(x)^{p} \psi\left(\mu^{\wedge}(x)\right) d x<\infty
$$

Proof. Since $\mu^{\wedge}(x)=\mu^{\wedge}(-x)$, we may as well restrict attention to positive values of $x$. Define

$$
F(x)=\int_{0}^{2 x} \psi(t) t^{p-1} d t \quad(x \geqslant 0) .
$$

It is easy to see from (10) that $F$ is convex nondecreasing and that

$$
x^{p} \psi(x) \leqslant F(x) \leqslant(2 x)^{p} \psi(2 x) \quad(x \geqslant 0) .
$$

In order to obtain (9), we need only show that $F\left(\mu^{\wedge}(x)\right)$ is integrable over [0, [. Moreover

$$
\int_{0}^{2 n \pi} F\left(\mu^{\wedge}(x)\right) d x \leqslant \int_{0}^{2 \pi} \sum_{w \in W_{n}} F\left(P_{n}(x+w)\right) d x .
$$

Accordingly the task is reduced to showing that (8) implies the uniform boundedness of

$$
S_{n}=\sum_{w \in W_{n}} F\left(P_{n}(x+w)\right) \text {. }
$$

Arguing as in the proof of the previous lemma and using convexity of $F$, we see that

$$
S_{n} \leqslant \sum_{w \in W_{n-1}} F\left(P_{n-1}(x+w)\right)+F\left(r_{n} P_{n-1}(x+w)\right) .
$$

However (12) and (11) combine to give

$$
S_{n}-S_{n-1} \leqslant \sum_{w \in W_{n-1}}\left(2 r_{n}\right)^{p} \psi\left(2 r_{n} P_{n-1}(x+w)\right) P_{n-1}(x+w)^{p} .
$$

Because $P_{n-1}(x+w)$ does not exceed one and $\psi$ is nondecreasing we deduce that

$$
S_{n}-S_{n-1} \leqslant \sum_{w \in W_{n-1}} 2^{p} \psi\left(2 r_{n}\right) r_{n}^{p} P_{n-1}(x+w)^{p},
$$

and the proof may now be completed by an application of Lemma 2 and the hypothesis (8). 
Although the formula $\mu=* \mu_{n}$ allows us to regard $\mu$ as the distribution of the sum $\sum X_{n}$ of independent random variables, with distribution $\mu_{n}$, we cannot apply the standard theorems in a useful way. This is because the canonical map from $\mathbf{R}^{\omega}$ (on which $\Sigma X_{n}$ is defined) to $\mathbf{R}$ loses information in this particular case. In plainer (but looser) language the $n$th digit of the binary expansion of a typical element of the support of $\mu$ is not determined by $\mu_{n}$ alone. This forces us to use Proposition 2, or something of the sort, but the next lemma guarantees that the added difficulty is not large.

LEMMA 4.

$$
\sum_{k=1}^{\infty} \sup _{n}\left|\mu^{\wedge}\left(2^{n} \pi+2^{n+k} \pi\right)-\mu^{\wedge}\left(2^{n} \pi\right) \mu^{\wedge}\left(2^{n+k} \pi\right)\right|<\infty .
$$

Proor. If $u_{j}, v_{j}, w_{j}$ all have modulus not greater than one, then

$$
\left|\Pi u_{j}-\Pi v_{j} \Pi w_{j}\right| \leqslant \Sigma\left|u_{j}-v_{j} w_{j}\right| \leqslant \Sigma\left|u_{j}-v_{j}\right|+\Sigma\left|1-w_{j}\right| .
$$

We see therefore that

$$
\begin{aligned}
\mid \mu^{\wedge}\left(2^{n} \pi+2^{n+k} \pi\right) & -\mu^{\wedge}\left(2^{n} \pi\right) \mu^{\wedge}\left(2^{n+k} \pi\right) \mid \\
& \leqslant \sum_{j=1}^{\infty}\left|\mu_{j}^{\wedge}\left(2^{n} \pi+2^{n+k} \pi\right)-\mu_{j}^{\hat{j}}\left(2^{n} \pi\right) \hat{\mu_{j}}\left(2^{n+k} \pi\right)\right| \\
& =\sum_{j=n+k}^{\infty}\left|\hat{\mu_{j}}\left(2^{n} \pi+2^{n+k} \pi\right)-\mu_{j}^{\hat{j}}\left(2^{n} \pi\right) \hat{\mu_{j}}\left(2^{n+k} \pi\right)\right| \\
& \leqslant \sum_{j=n+k}^{\infty}\left|\mu_{j}^{\hat{j}}\left(2^{n} \pi+2^{n+k} \pi\right)-\mu_{j}^{\hat{j}}\left(2^{n+k} \pi\right)\right|+\sum_{j=n+k}^{\infty}\left|1-\mu_{j}^{\hat{j}}\left(2^{n} \pi\right)\right| .
\end{aligned}
$$

Writing $M_{k}$ for the majorant just found, and changing the summing index to $i=j-n-k$, we have

$$
\begin{aligned}
M_{k} \leqslant \sum_{i=0}^{\infty}\left\{\left|\cos ^{2}\left(s_{i}+t_{i}\right)-\cos ^{2}\left(s_{i}\right)\right|+\mid \sin ^{2}\left(s_{i}\right.\right. & \left.\left.+t_{i}\right)-\sin ^{2}\left(s_{i}\right) \mid\right\} \\
& +\sum_{i=0}^{\infty}\left\{\left|1-\cos ^{2}\left(t_{i}\right)\right|+\sin ^{2}\left(t_{i}\right)\right\},
\end{aligned}
$$

where $s_{i}=2^{-1-i} \pi$ and $t_{i}=2^{-k-1-i} \pi$.

It is now easy to see that $\sum_{k=1}^{\infty} M_{k}$ is finite, as required.

Proof of MAIN THeOReM fOR THE LINe (AND CIRCLE). Suppose that $p \in[1, \infty[$ has been given together with the function $\varphi$. We choose $\sigma_{p}, \tau_{p}$ to be measures $\mu$ of the type discussed in this section, taking $r_{n}=s_{n}$ (given by Proposition 1(i)) for $\mu=\sigma_{p}$, and $r_{n}=t_{n}$ (given by Proposition 1(ii) )for $\mu=\tau_{p}$. Before we determine the choice more precisely we note that this process must lead in any case to an ergodic probability measure whose transform lies in $C_{0}^{+}(R)$ and whose support is $[-1,1]$. 
Let us consider $\sigma_{p}$ first. Guided by Lemma 1 , we specify the sequence $\left(a_{n}\right)$, appearing in Proposition 1, to be $a_{n}=4^{1-n}$. We note further that there is no loss of generality in supposing that $\varphi$ is decreasing in the wide sense and demanding that $\varphi$ approaches $\infty$ so slowly that $\sum s_{n}^{r}=\infty$ whenever $r<p$. (We can, for example, replace $\varphi$ by $\Phi$ as in the proof of Proposition 1.) Let us assume then that $\left(s_{n}\right)$ has been chosen according to these rules and immediately relabel $\left(s_{n}\right)$ and $\sigma_{p}$ by $\left(r_{n}\right)$ and $\mu$, respectively, so that we may apply the lemmas of this section. The first observation is that Lemma 2 ensures finiteness of $\int \mu^{\wedge}(x)^{p} d x$. In fact the argument parallels part of the proof of Lemma 3 , since we simply note that

$$
\int_{0}^{2^{n} \pi} \mu^{\wedge}(x)^{p} d x \leqslant \int_{0}^{2 \pi} \sum_{w \in W_{n}} P_{n}(x+w)^{p} d x \leqslant 2 \pi \prod_{j=1}^{\infty}\left(1+r_{j}^{p}\right)
$$

The other integral estimate follows from Lemma 1 . In fact

$$
\begin{aligned}
\int \mu^{\wedge}(x)^{p} \varphi\left(\mu^{\wedge}(x)\right) d x & \geqslant \sum_{n=1}^{\infty} \int_{-\pi}^{\pi} \mu^{\wedge}\left(x+2^{n} \pi\right)^{p} \varphi\left(\mu^{\wedge}\left(x+2^{n} \pi\right)\right) d x \\
& \geqslant \sum_{n=1}^{\infty} A^{p} r_{n}^{p} \varphi\left(r_{n}+4^{1-n}\right) \geqslant A^{p} \sum_{n=1}^{\infty} r_{n}^{p} \varphi\left(2 r_{n}\right),
\end{aligned}
$$

because $\varphi$ is nonincreasing. It now follows from Proposition 1(i) that

$$
\int \mu^{\wedge}(x)^{p} d x<\infty, \int \mu^{\wedge}(x)^{p} \varphi\left(\mu^{\wedge}(x)\right) d x=\infty .
$$

It remains to consider the comparability of $\mu$ and Lebesgue measure. The easy case is when $p \leqslant 2$ because then $\Sigma r_{n}^{2}<\infty$, and the lack of independence operates in our favour to ensure the appropriate absolute continuity properties. The point is that $m$, the restriction of Lebesgue measure to $[-1,1]$, can be expressed as the weak * limit, $m=*_{n=1}^{\infty} m_{n}$, where $m_{n}=\frac{1}{2} \delta(0)+\frac{1}{4}\left(\delta\left(2^{-n}\right)+\delta\left(-2^{-n}\right)\right.$ ). (In other words, $m$ is the special case of $\mu$ with $r_{n} \equiv 0$.) It is easy to see from Proposition 3 that the product measure $\otimes \mu_{n}$ belongs to $L_{r}\left(\otimes m_{n}\right)$ for $r \geqslant 1$ (hence $r>0$ ), and a fortiori $\mu$ belongs to $L_{r}(m)$. For a direct argument, one may note that, for $r \geqslant 1$,

$$
\int\left(d \pi_{n}^{\prime} / d p_{n}^{\prime}\right)^{r} \leqslant \int\left(d \pi_{n} / d p_{n}\right)^{r} d p_{n} \leqslant \prod_{j=1}^{n}\left[\frac{1}{2}\left(1+r_{j}\right)^{r}+\frac{1}{2}\left(1-r_{j}\right)^{r}\right],
$$

where

$$
\begin{aligned}
\pi_{n}=\mu_{1} \otimes \ldots \otimes \mu_{n}, p_{n}=m_{1} \otimes \ldots \otimes m_{n}, \pi_{n}^{\prime} & =\mu_{1} * \ldots * \mu_{n} * m_{[0,2-n]}, \\
p_{n}^{\prime} & =m_{1} * \ldots * m_{n} * m_{[0,2-n]} .
\end{aligned}
$$

This is the sort of argument used in Saeki (1977).

When $p>2$ then our choice ensures that $\sum r_{n}^{2}=\infty$, and we must deduce that $\mu$ is singular to Lebesgue measure. In this case the lack of independence tends to work against us but we can appeal to Proposition 2 with the choice

$$
X_{n}(x)=\exp \left(2^{n} \pi i x\right)
$$


$P_{1}=\mu, P_{2}$ is Lebesgue measure. Lemma 4 ensures (i) for expectation with respect to $P_{1}$ and the corresponding formula for $E_{2}$ is trivial in view of orthogonality. The same orthogonality reduces (ii) of Proposition 2 to the statement

$$
\sum_{n=1}^{\infty} \mu^{\wedge}\left(2^{n} \pi\right)^{2}=\infty
$$

and, of course, this follows from Lemma 1 and the fact that $\left(r_{n}\right)$ is not square summable. We have now verified all the assertions concerning the measure $\sigma_{p}$.

In choosing $\tau_{p}$, we find it convenient to relabel the reciprocal of $\varphi(x)$ as $\psi(x)$ and assume that $\psi$ is nondecreasing. In addition we ensure as before that $\sum_{n=1}^{\infty} t_{n}^{r}<\infty$, whenever $r<p$. Then we take $\tau_{p}$ to be $\mu$ with $r_{n} \equiv t_{n}$. Since we have ensured that $\sum r_{n}^{2}<\infty$ if and only if $p<2$, arguments identical with those just given for $\sigma_{p}$ show that $\tau_{p}$ is singular for $p \geqslant 2$ and has a Radon-Nikodym derivative (with respect to Lebesgue measure) in $\bigcap_{r>0} L_{r}$ when $p<2$. Accordingly we need only address our attention to the integral estimates given in (ii) of the theorem. The second of these is nothing more than Lemma 3, so we need only check that, in the case presently under discussion,

$$
\int \mu^{\wedge}(x)^{p} d x=\infty .
$$

Because $\sum r_{n}^{p}=\infty$ and $\mu^{\wedge}$ is nonnegative this is an immediate consequence of Lemma 1 , so the theorem is proved for the line. It is clear that virtually the same arguments give the corresponding result for the circle.

\section{General LCA groups}

(4.1) Products of cyclic groups. In this subsection we prove the main theorem for the case where $G=\Pi_{n=1}^{\infty} Z\left(m_{n}\right)$, where $\left(m_{n}\right)$ is an arbitrary sequence of positive integers greater than or equal to two. (It is the possibility $m_{n} \rightarrow \infty$ which prevents us from dismissing this case in a few words.)

As in the last section we take a parameter sequence $\left(r_{n}\right)$ of positive real numbers which do not exceed one and focus attention on measures $\mu$ which are infinite convolutions of discrete factors, each determined by a single $r_{n}$. Formally we write

$$
\begin{gathered}
\mu=\stackrel{\substack{* \\
n=1}}{*} \mu_{n}, \\
\mu_{n}=m_{m}^{-1}\left[\left(1+\left(m_{n}-1\right) r_{n}\right) \delta(0)+\Sigma^{\prime}\left(1-r_{n}\right) \delta(g)\right],
\end{gathered}
$$

where $g$ is summed over $\mathbf{Z}\left(m_{n}\right) \backslash\{0\}$. Observe that, if we allowed the special case where $r_{n} \equiv 0$, then $\mu$ would equal Haar measure $\lambda$ on $G$. It is a convenience to introduce the additional notation $G_{n}$ as an alternative to $Z\left(m_{n}\right)$, to write $\lambda_{n}$ for Haar measure on $G_{n}$, and to write $X_{n}=G^{\wedge}$ for the dual group. 
A typical character $\chi$ in $X$ is a finite product, $\chi_{n(1)} \chi_{n(2)} \ldots \chi_{n(k)}$, where $\chi_{n(i)} \in X_{n(i)}, i=1, \ldots, k$. If each $\chi_{n(i)}$ is non-trivial then we have

$$
\mu^{\wedge}(\chi)=\prod_{i=1}^{k} r_{n(i)}
$$

(The simplest way to derive (3) from (1), (2) is to note that $\mu_{n}=r_{n} \delta(0)+\left(1-r_{n}\right) \lambda_{n}$.) It is now obvious, under our restriction that $\left.\left.r_{n} \in\right] 0,1\right]$, that $\mu$ is an ergodic probability measure whose transform belongs to $C_{0}^{+}(X)$ if and only if $r_{n} \rightarrow 0$. We shall, of course, demand that $r_{n} \rightarrow 0$, in which case we clearly have the further property that the support of $\mu$ is $G$ itself.

Let us now consider, on the measure space $\left(G, \otimes \lambda_{n}\right)$, the random variables $X_{n}$ corresponding to $d \mu_{n} / d \lambda_{n}$. Note that

$$
E\left(X_{n}^{r}\right)=m_{n}^{-1}\left[\left(1+\left(m_{n}-1\right) r_{n}\right)^{r}+\left(1-r_{n}\right)^{r}\left(m_{n}-1\right)\right] .
$$

We see from (4) that $E\left(X_{n}\right)=1$ and that $\Pi E\left(X_{n}^{r}\right)$ converges for $r>1$ when $\sum_{n=1}^{\infty}\left(m_{n}-1\right) r_{n}^{2}<\infty$. It follows from Proposition 3 that $d \mu / d \lambda \in \bigcap_{r>1} L_{r}(G)$ when the latter hypothesis holds. On the other hand, we may also apply Proposition 3 (with $r=2$ and $X_{n}$ replaced by $X_{n}^{\ddagger}$ ) together with (4) for $r=\frac{1}{2}$ to see that $\mu$ is singular if that hypothesis does not hold. In fact we can prove the following:

LEMMA 5. If $\sum_{n=1}^{\infty}\left(m_{n}-1\right) r_{n}^{2}<\infty$ then $\mu$ is absolutely continuous with respect to $\lambda$, and $d \mu / d \lambda \in \bigcap_{p>0} L_{p}(G)$. Otherwise $\mu$ is singular to $\lambda$.

Let us now suppose that $p \in[1, \infty[$ and $\varphi$, as in the statement of the main theorem, are given. Choose as in Section 3 sequences $\left(s_{n}\right),\left(t_{n}\right)$ as guaranteed by Proposition 1 and take $\sigma_{p}$ to be the measure $\mu$ with parameters $r_{n}=s_{n}\left(m_{n}-1\right)^{1 / p} ; \tau_{p}$ to be the measure $\mu$ with parameters $r_{n}=t_{n}\left(m_{n}-1\right)^{-1 / p}$.

Observe that, for $p \geqslant 2$, we have $r_{n}^{2}\left(m_{n}-1\right) \geqslant s_{n}^{2}$ or $t_{n}^{2}$ as the case may be. Hence $\sigma_{p}$ is singular for $p>2$ and $\tau_{p}$ is singular for $p \geqslant 2$, in view of Lemma 5 . The appropriate statements about the Radon-Nikodym derivatives of $\sigma_{p}, p \leqslant 2$, and $\tau_{p}, p<2$, also follow simply from Lemma 5 .

We complete the proof of the theorem for $G=\prod_{p=1}^{\infty} Z\left(m_{n}\right)$ by verifying the integral formulae (i), (ii) in the statement. In what follows it will be convenient to use the symbol $\Sigma_{n}$ to denote summation over those characters which are trivial on each of $G_{n}, G_{n+1}, \ldots$. In fact we deduce from (3) that

$$
\Sigma_{n} \mu^{\wedge}(\chi)^{p}=\prod_{j=1}^{n}\left(1+\left(m_{j}-1\right) r_{j}^{p}\right) .
$$

Of course it follows immediately from (5) that

$$
\int \sigma_{p}(\chi)^{p} d \theta(\chi)<\infty, \quad \int \tau_{p}(\chi)^{p} d \theta(\chi)=\infty
$$


To obtain the remaining inequalities we elaborate (5). In fact let us write

$$
S_{n}=\Sigma_{n} \mu^{\wedge}(\chi)^{p} \psi\left(\mu^{\wedge}(\chi)\right),
$$

where $\psi$ is nondecreasing as before. We have

$$
S_{n}-S_{n-1} \leqslant\left(m_{n}-1\right) r_{n}^{p} \psi\left(r_{n}\right) \prod_{j=1}^{n-1}\left(1+\left(m_{j}-1\right) r_{j}^{p}\right) .
$$

To check (8), note that

$$
\begin{aligned}
\Sigma_{n} \mu^{\wedge}(\chi)^{p} \psi\left(\mu^{\wedge}(\chi)\right) & =\Sigma_{n-1}\left(\mu^{\wedge}(\chi)^{p} \psi\left(\mu^{\wedge}(\chi)\right)+\left(m_{n}-1\right) r_{n}^{p} \mu^{\wedge}(\chi)^{p} \psi\left(r_{n} \mu^{\wedge}(\chi)\right)\right) \\
& \leqslant \Sigma_{n-1} \mu^{\wedge}(\chi)^{p} \psi\left(\mu^{\wedge}(\chi)\right)+\left(m_{n}-1\right) r_{n}^{p} \psi\left(r_{n}\right) \Sigma_{n-1} \mu^{\wedge}(\chi)^{p},
\end{aligned}
$$

and use (5).

In the special case where $\mu=\tau_{p}$ we have $r_{n}=t_{n}\left(m_{n}-1\right)^{-1 / p}$, so that (8) yields

$$
S_{n}-S_{n-1} \leqslant \psi\left(2 t_{n}\right) t_{n}^{p} \prod_{j=1}^{n-1}\left(1+t_{j}^{p}\right)
$$

Taking (9) together with (ii) of Proposition 1, we deduce that

$$
\int \tau_{p}(\chi)^{p} \psi\left(\tau_{p}(\chi)\right) d \theta(\chi)<\infty .
$$

Replacing $\psi$ by the non-increasing function $\varphi$ and reversing all the inequalities in the proof of $(8)$, we find

$$
S_{n}-S_{n-1} \geqslant\left(m_{n}-1\right) r_{n}^{p} \varphi\left(r_{n}\right) \prod_{j=1}^{n-1}\left(1+\left(m_{j}-1\right) r_{j}^{p}\right) .
$$

In fact, when $\mu=\sigma_{p}$, all we need from (11) is the statement that

$$
S_{n}-S_{n-1} \geqslant s_{n}^{p} \varphi\left(2 s_{n}\right),
$$

because this leads via (i) of Proposition 1 to the estimate

$$
\int \sigma_{p}^{\wedge}(\chi)^{p} \varphi\left(\sigma_{p}(\chi)\right) d \theta(\chi)=\infty .
$$

The combination of (6), (10) and (13) completes the proof for the groups presently under consideration.

(4.2) The q-adic integers. Here we prove the main theorem for the case where $G=\Delta_{q}$, where $q$ is a prime. In other words, we have

$$
G=\lim _{\leftarrow} \mathbf{Z}\left(q^{n}\right), \quad X=\underline{\lim } \mathbf{Z}\left(q^{n}\right)^{\wedge}
$$

where ${ }^{\wedge}$ denotes dual, and

$$
G / q^{n} G \simeq \mathbf{Z}\left(q^{n}\right), \quad q^{n} G=\left\{q^{n} x: x \in G\right\}
$$


In fact we write $X_{n}$ for $\mathrm{Z}\left(q^{n}\right)^{\wedge}$, regard this as a subgroup of $X$ and write $\theta_{n}$ for counting measure on $X_{n}$. We write elements of $X_{n}$ as rational numbers, so that

$$
X_{n}=\left\{\sum_{i=0}^{n-1} k_{i} q^{-i}: k_{i} \in\{0,1, \ldots, q-1\}\right\},
$$

and elements of $G$ as formal sums of the form

$$
x=\sum_{i=0}^{\infty} x_{i} q^{i}
$$

where $x_{i} \in\{0,1, \ldots, q-1\}$. In particular, if $\chi \in X_{n}$ and $x$ in $G$ is given by (17), we have

$$
\chi(x)=e\left(\chi \sum_{i=0}^{m} x_{i} q^{i}\right), \text { for all } m \text { sufficiently large. }
$$

Although the first statement in (14) appears unhelpful because all our methods relate to direct limits we avoid the problem by working on a dense countable subgroup of $G$ which can be realized in a useful way as a direct limit (though not in the category of groups). The group in question is generated by all finite sums appearing in (17).

Once again we take a parameter sequence $\left(r_{n}\right)$ of positive real numbers not exceeding one, and work with an infinite convolution, $\mu=* \mu_{n}$, where each $\mu_{n}$ depends only on $r_{n}$. In fact we define the measure $\rho_{n}$ on $G$, by

$$
\rho_{n}=q^{-1} \sum_{i=0}^{q-1} \delta\left(i q^{n-1}\right)
$$

and set

$$
\mu_{n}=\left(1-r_{n}\right)\left(\rho_{n} * \tilde{\rho}_{n}\right)+r_{n} \delta(0) .
$$

It is of some interest to note that this is the same pattern as used in both Section 3 and (4.1). (Observe that, in (4.1), $\lambda_{n} * \lambda_{n}^{\sim}=\lambda_{n}$.)

From (16), (19) and (20) we see that $\mu_{n}$ is determined on $X_{n}$, by

$$
\mu_{n}^{\hat{n}}\left(\sum_{i=0}^{n-1} k_{i} q^{-i}\right)= \begin{cases}r_{n}, & \text { if } k_{n-1} \neq 0, \\ 1, & \text { if } k_{n-1}=0 .\end{cases}
$$

It is now clear that, when $r_{n} \rightarrow 0, \mu$ is an ergodic probability measure with full support such that $\mu^{\wedge} \in C_{0}^{+}(X)$. However, we shall require estimates of the transform of $\mu$ which are more far-reaching than (21). Accordingly let us note for future reference that

$$
\left(\rho_{n-m+1}\right)^{\wedge}\left(a q^{-n}\right)=q^{-1}\left(1-e\left(a q^{1-m}\right)\right)\left(1-e\left(a q^{-m}\right)\right)^{-1},
$$

where $a, n, m$ are positive integers, $n \geqslant m$, and the right-hand side is taken to be zero when $q^{m}$ is a factor of $a$.

As before we assume that $\varphi$ is nonincreasing and, for each $p$, choose sequences $\left(s_{n}\right),\left(t_{n}\right)$ as guaranteed by Proposition 1. In this case we may as well set $a_{n} \equiv 0$ 
in (i) of the statement of that proposition, but we take $C=q$ in (ii). Once again $\sigma_{p}$ is obtained by setting $r_{n}=s_{n}$ in the definition of $\mu$ and $\tau_{p}$ is obtained by setting $r_{n}=t_{n}$ in that definition.

The rest of the proof parallels the discussion in Section 3, so let us set about obtaining an analogue of Lemma 1. Observe, from (21) and the definition of $\mu$, that for every positive integer $n \geqslant 2$,

$$
\mu \wedge\left(q^{1-n}\right)=r_{n} \prod_{j=1}^{n-1}\left(1-\left(1-r_{j}\right)\left(1-\left|\hat{\rho_{j}}\left(q^{1-n}\right)\right|^{2}\right)\right) .
$$

From (22), (23), we see, in particular, that

$$
\mu^{\wedge}\left(q^{1-n}\right) \geqslant r_{n} \prod_{k=2}^{\infty}\left(\left|\left(1-e\left(q^{1-k}\right)\right) /\left(1-e\left(q^{-k}\right)\right)\right|^{2} q^{-2}\right) .
$$

The only information we require from (23) and (24) is the conclusion that there exists a positive constant $A$ (independent of $n$ ) such that

$$
r_{n} \geqslant \mu^{\wedge}\left(q^{1-n}\right) \geqslant A r_{n}, \quad n \geqslant 2 ;
$$

and it is a simple matter to deduce from (25) that

$$
\int \tau_{p}(\chi)^{p} d \theta(\chi)=\infty, \quad \int \hat{\sigma_{p}}(\chi)^{p} \varphi\left(\sigma_{p}(\chi)\right) d \theta(\chi)=\infty .
$$

The remaining integrability assertions in (i), (ii) of the theorem for $G=\Delta_{q}$ will follow from an analogue of Lemmas 2 and 3, which we now establish.

LEMma 6. Suppose that $F:[0, \infty] \rightarrow[0, \infty]$ is nondecreasing and convex. Then

$$
\int_{X_{n}} F\left(\mu^{\wedge}(\chi)\right) d \theta_{n}(\chi)-\int_{X_{n-1}} F\left(\mu^{\wedge}(\chi)\right) d \theta_{n-1}(\chi) \leqslant q \int_{X_{n-1}} F\left(r_{n} \mu^{\wedge}(\chi)\right) d \theta_{n-1}(\chi) .
$$

Proor. Note first that, for any $\chi \in X$ and any positive constant $B$,

We may write

$$
\begin{aligned}
F\left(\mu_{n}^{\hat{n}}(\chi) B\right) & =F\left(\left(1-\left|\hat{\rho_{n}}(\chi)\right|^{2}\right) r_{n} B+\left|\hat{\rho_{n}}(\chi)\right|^{2} B\right) \\
& \leqslant\left(1-\left|\hat{\rho_{n}}(\chi)\right|^{2}\right) F\left(r_{n} B\right)+\left|\rho_{n}^{\hat{n}}(\chi)\right|^{2} F(B) .
\end{aligned}
$$

$$
X_{n}=\left\{x+k q^{1-n}: \chi \in X_{n-1}, k=0,1, \ldots, q-1\right\},
$$

and note that, for each $\chi$ in $X_{n-1}$,

$$
\sum_{k=0}^{q-1}\left|\hat{\rho_{n}}\left(\chi+k q^{1-n}\right)\right|^{2} \leqslant 1
$$

(To check (29) consider the measure induced by $\chi \cdot \rho_{n}$ on $\mathbf{Z}(q)$ and apply Parseval's formula.)

Now, for each $\chi$ belonging to $X_{n-1}$, let $B=B(\chi)=\mu^{\wedge}(\chi)$, recalling from (21) that $B(\chi)$ is independent of $r_{m}, m>n-1$. Using (27) and (29) we find, for each $\chi$ 
in $X_{n-1}$,

$$
\begin{aligned}
\sum_{k=0}^{q-1} F\left(\mu_{n}^{\hat{\prime}}\left(\chi+k q^{1-n}\right)\right) & =\sum_{k=0}^{q-1} F\left(\mu_{n}^{\hat{n}}\left(\chi+k q^{1-n}\right) B(\chi)\right) \\
& \leqslant q F\left(r_{n} B(\chi)\right)+F(B(\chi)),
\end{aligned}
$$

and, in view of (28), we may complete the proof of the lemma by summing both sides of (30) with respect to $\chi$.

It is a simple consequence of Lemma 6 that, for $p \geqslant 1$,

$$
\int \mu^{\wedge}(\chi)^{p} d \theta_{n}(\chi) \leqslant \prod_{j=1}^{n}\left(1+q r_{j}^{p}\right) .
$$

Moreover, by choosing $F$ as in Section 3, we can obtain from (31) and Lemma 6 the estimate

$$
\int \mu^{\wedge}(\chi)^{p} \psi\left(\mu^{\wedge}(\chi)\right) d \theta_{n}(\chi) \leqslant C+\sum_{j=2}^{n} q r_{j}^{p} \psi\left(2 r_{j}\right) \prod_{k=1}^{j-1}\left(1+q r_{k}^{p}\right),
$$

where $C$ is some positive constant. Of course (31) and (32) ensure the remaining estimates required for (i), (ii) of the theorem.

To complete the proof for $G=\Delta_{q}$ we need only check the analogue of Lemma 5 with $m_{n}=q$ (or, for that matter, any constant greater than one). Let us write $\lambda_{n}=\rho_{n} * \tilde{\rho}_{n}$ and note that $*_{n=1}^{\infty} \lambda_{n}$ equals $\lambda$, the Haar measure of $G$. That makes it obvious that the arguments of Lemma 5 would be effective if we could replace the convolution operation by the measure product operation. In fact this is not possible but we can certainly handle the absolutely continuous case in the manner used for the corresponding problem on $\mathbf{R}$. (Here the auxiliary tail measure, corresponding to $m_{[0,2-n]}$ in the previous argument, is Haar measure on $q^{n} G$.) In the singular case we appeal once more to Proposition 2 , and take $P_{1}=\lambda, P_{2}=\mu$. Evidently we should choose the random variable $X_{n}(t)$ to be $e\left(q^{-n} t\right)$, because (ii) of Proposition 2 is then obvious from (25) and orthogonality. Thus the final step of the verification of the case where $G=\Delta_{q}$ becomes the next lemma.

\section{LEMMA 7.}

$$
\sum_{k=0}^{\infty} \sup _{n}\left|\mu^{\wedge}\left(q^{-n}+q^{-n-k}\right)-\mu^{\wedge}\left(q^{-n}\right) \mu^{\wedge}\left(q^{-n-k}\right)\right|<\infty
$$

ProOf. It is a convenience to introduce the function $g$, defined by

and to write

$$
g(x)=\sin (\pi q x) / q \sin (\pi x), \quad 0<x<1,
$$

$$
G(m, k)=2\left|g\left(q^{-m-k}+q^{-m}\right)-g\left(q^{-m}\right)\right|+2\left|g\left(q^{-m-k}\right)-1\right| .
$$


In fact, we show first that, for $n=1,2, \ldots, k=1,2, \ldots$,

$$
\left|\mu^{\wedge}\left(q^{-n}+q^{-n-k}\right)-\mu^{\wedge}\left(q^{-n}\right) \mu^{\wedge}\left(q^{-n-k}\right)\right| \leqslant \sum_{m=1}^{\infty} G(m, k) .
$$

(Observe that the right-hand side of (35) is independent of $n$.)

The first step in proving (35) is to write the left-hand side as an infinite product, $\Pi\left|u_{j}-v_{j} w_{j}\right|$, where $u_{j}=\mu_{j}\left(q^{-n}+q^{-n-k}\right), v_{j}=\mu_{j}\left(q^{-n}\right), w_{j}=\mu_{j}\left(q^{-n-k}\right)$. Then the quantity to be estimated becomes $\Sigma\left|u_{j}-v_{j}\right|+\Sigma\left|1-w_{j}\right|$, where both sums are actually finite. In fact we have from (19) and (20) the estimates

$$
\begin{aligned}
& \left|u_{j}-v_{j}\right| \leqslant 2\left|\rho_{j}^{\hat{j}}\left(q^{-n}+q^{-n-k}\right)-\rho_{j}^{\hat{j}}\left(q^{-n}\right)\right|, \\
& \left|1-w_{j}\right| \leqslant 2\left|\rho_{j}^{\hat{j}}\left(q^{-n-k}\right)-1\right| .
\end{aligned}
$$

Summing (36) and (37) in the reverse direction and applying (22) we arrive at (35).

To complete the proof of the lemma we must show that the sum of $G(m, k)$ over all positive integers $m$ and $k$ is finite. There is no difficulty with the second term on the right-hand side of (34), because $|1-g(x)|=O\left(x^{2}\right)$ as $x \rightarrow 0$. In similar vein we note that for $0<y<x,|g(x+y)-g(x)|=O(x y)$, as $x \rightarrow 0$, so the result will follow as soon as we have proved that, for fixed $m$,

$$
\sum_{k=1}^{\infty}\left|g\left(q^{-m-k}+q^{-m}\right)-g\left(q^{-m}\right)\right|<\infty
$$

However it is easy to see (for example by the Mean Value Theorem) that the summand in (38) does not exceed $2 \pi q^{-k}$, and this proves the lemma.

(4.3) Combination of cases. Now that we have proved the theorem for all the special cases enumerated in (2.4) it remains to show that Proposition 4 can be applied to give the general result. We merely sketch the (standard) arguments.

Suppose first that the theorem holds for the group $H$ and that $H$ is an open subgroup of $G$. We simply regard the measures on $H$ as measures on $G$, and note that the annihilator, $H^{\perp}$, of $H$ is a compact subgroup of $X$. Weil's formula then ensures the validity of all the statements concerning the transforms (including the fact that the transforms vanish at infinity).

Now consider the case where the theorem holds for the group $G / K$ where $K$ is a compact subgroup of $G$. Writing, as usual, $\mu$ to stand for either $\sigma_{p}$ or $\tau_{p}$, we lift $\mu$ to the (unique) measure $\nu$ in $M(G) * \lambda_{K}$ such that $\pi\left({ }_{K}^{*} \nu\right)=\mu$. (This process is discussed in Reiter $(1968,8.2 .7)$, and Brown $(1973,2.5)$.) In other words,

$$
\int_{G / K} f(t) d \mu(t)=\int_{G} f_{\circ} \pi_{K}(x) d v(x),
$$

where $f$ runs through continuous functions on $G / K$ with compact support, and $\pi_{K}: G \rightarrow G / K$ is the canonical projection. Because the Haar measure $\lambda_{K}$ of $K$ is a 
factor of $\nu$, we see that $\nu^{\wedge}(\chi)$ is zero unless $\chi$ belongs to $K^{\perp}$, in which case $\chi=\chi^{\prime} \circ \pi_{K}$, for some $\chi^{\prime}$ in $(G / K)^{\wedge}$. It is then clear from (39) that $\nu^{\wedge}(\chi)$ equals $\mu^{\wedge}\left(\chi^{\prime}\right)$ and the several assertions concerning the Fourier-Stieltjes transforms follow. We have defined ergodicity in such a way that $\nu$ is ergodic. Moreover $\pi_{K}^{*}: \lambda_{K} * M(G) \rightarrow M(G / K)$ is an isometric $\sim$-algebra isomorphism so that $\nu$ is singular. The statement concerning the Radon-Nikodym derivative of $\nu$ can be deduced from Weil's formula, that is, the analogue of (39) which links the Haar measures.

Finally, let us consider the case where the theorem holds for a group $G_{1}$ and we require it for the product, $G=G_{1} \times G_{2}$. The argument is similar to (9.11) of Hewitt and Ritter (1977) and what we need is an auxiliary measure $\rho$ on $G_{2}$. We require that $\rho$ be an ergodic probability measure whose support is a compact neighbourhood of zero and whose Radon-Nikodym derivative with respect to Haar measure, $\lambda_{2}$, belongs to $\bigcap_{r>0} L_{r}\left(G_{2}\right)$. Further we insist that $\rho^{\wedge} \geqslant 0$ and that

$$
\int_{X_{2}} \rho^{\wedge}(\chi)^{p} d \theta_{2}(\chi)<\infty
$$

(Despite such a formidable list of demands, $\rho$ is rather easy to find. For example, if $G_{2}$ is compact then we may take $\rho=\lambda_{2}$ !) Yet again using $\mu$ to serve for $\sigma_{p}$ or $\tau_{p}$ on $G_{1}$, we define $\nu$ on $G$ as the product measure, $\mu \otimes \rho$. The various properties are easily checked. In particular, note that

$$
\begin{aligned}
& \int \nu^{\wedge}(\chi)^{p} \varphi\left(\nu^{\wedge}(\chi)\right) d \theta(\chi) \geqslant \int \rho^{\wedge}\left(\chi_{2}\right)^{p} d \theta_{2}\left(\chi_{2}\right) \int \mu^{\wedge}\left(\chi_{1}\right)^{p} \varphi\left(\mu^{\wedge}\left(\chi_{1}\right)\right) d \theta_{1}\left(\chi_{1}\right), \\
& \int \nu^{\wedge}(\chi)^{p} \psi\left(\nu^{\wedge}(\chi)\right) d \theta(\chi) \leqslant \int \rho^{\wedge}\left(\chi_{2}\right)^{p} d \theta_{2}\left(\chi_{2}\right) \int \mu^{\wedge}\left(\chi_{1}\right)^{p} \psi\left(\mu^{\wedge}\left(\chi_{1}\right)\right) d \theta_{1}\left(\chi_{1}\right),
\end{aligned}
$$

where $\varphi$ is nonincreasing, $\psi$ is nondecreasing.

(4.4) Ergodic measures. Although it is not a strictly necessary part of the proof of the theorem as stated, it remains to show that our use of the word "ergodic" is reasonable. We made the definition in such a way that the canonical measure obtained by lifting a $D$-ergodic measure from $G / K, K$ compact, to $G$ is again called ergodic. Now suppose that we consider a different compact subgroup $H$ of $G$ and take the canonical quotient of our lifted measure. If $G / H$ is a group on which we have an explicit realization of Haar measure as an infinite convolution (for example, all the special groups considered above) then we would certainly expect that the quotient measure under discussion should be $D$-ergodic for a suitable choice of $D$. The next proposition shows this in an explicit way. 
Proposition 5. Let $G$ be a locally compact abelian group and suppose that $H, K$ are compact subgroups of $G$. Suppose further that $\mu$ is a probability measure on $G$ and that

(i) $\pi_{K}^{*}(\mu)$ is $D_{1}$-ergodic in $M(G / K)$,

(ii) $\pi_{H}^{*}\left(\lambda_{K}\right)$ is $D_{2}$-ergodic in $M(G / H)$,

where $\lambda_{K}$ denotes Haar measure on $K$. Then, if $D$ is any countable subgroup of $G / H$ which contains $D_{2}$ and $\pi_{H}\left(C_{1}\right)$, where $C_{1}(\subseteq G)$ is a set of coset representatives for $D_{1}$,

(iii) $\pi_{H}^{*}\left(\mu * \lambda_{K}\right)$ is D-ergodic in $M(G / H)$.

Proof. We have

$$
\pi_{H}^{*}\left(\mu * \lambda_{K}\right)=\pi_{H}^{*}\left(\mu * \lambda_{K} * \lambda_{H}\right)=\pi_{H}^{*}\left(\mu * \lambda_{H}\right) \pi_{H}^{*}\left(\lambda_{K} * \lambda_{H}\right),
$$

and the problem is to show that

$$
\pi_{H}^{*}\left(\mu * \lambda_{K}\right)\left(E+D_{2}+\pi_{H}\left(C_{1}\right)\right)=1,
$$

whenever $E$ is a Borel set in $G / H$ which is not $\pi_{I I}^{*}\left(\mu * \lambda_{K}\right)$-null. In fact we see, from (40) and (41), that the problem may be reformulated as that of proving

$$
\pi_{H}^{*}\left(\mu * \lambda_{K}\right)\left(A+B+D_{2}+\pi_{H}\left(C_{1}\right)\right)=1,
$$

whenever $A$ and $B$ are Borel sets in $G / H$ such that $\pi_{H}^{*}(\mu)(A), \pi_{H}^{*}\left(\lambda_{K}\right)(B)$ are both non-zero. Let us choose a countable set $C_{2}$ of coset representatives of $D_{2}$ (with respect to $H$ ). Equation (42) becomes the requirement that

$$
\mu * \lambda_{K}\left(\pi_{H}^{-1}(A)+\pi_{H}^{-1}(B)+C_{2}+C_{1}\right)=1 .
$$

Writing $F$ for the set on which $\mu * \lambda_{K}$ is evaluated in (43), we note that, for all $g \in \pi_{H}^{-1}(A)+K+C_{1}$,

for some $k$ in $K$.

$$
F-g \supseteq \pi_{H}^{-1}(B)+C_{2}-k,
$$

By the translation invariance under $K$ of the Haar measure $\lambda_{K}$, we see from (44) that

$$
\lambda_{K}(F-g) \geqslant \lambda_{K}\left(\pi_{H}^{-1}(B)+C_{2}\right),
$$

with $g$ as previously restricted. However, $B$ is not $\pi_{H}^{*}\left(\lambda_{K}\right)$-null and $\pi_{H}^{*}\left(\lambda_{K}\right)$ is a $D_{2}$-ergodic measure. Therefore the right-hand side of (45) equals one. Accordingly

$$
\mu * \lambda_{K}(F)=\int \lambda_{K}(F-g) d \mu(g) \geqslant \mu\left(\pi_{H}^{-1}(A)+K+C_{1}\right) .
$$

However, the set $\pi_{H}^{-1}(A)+K+C_{1}$ contains $\pi_{H}^{-1}(A)$ and hence is not $\mu$-null (because $\pi_{H}^{*} \mu(A)>0$ ). Therefore the inequality (46) becomes

$$
\mu * \lambda_{K}(F) \geqslant \pi_{K}^{*} \mu\left(\pi_{K}\left(\pi_{H}^{-1}(A)\right)+D_{1}\right)=1,
$$

because $\pi_{K}^{*} \mu$ is $D_{1}$-ergodic.

Because (47) gives the desired equality (43) the proof is complete. 


\section{References}

R. P. Boas, Jnr. (1967), Integrability Theorems for Trigonometric Transforms (Springer-Verlag, Berlin, 1967).

G. Brown (1973), " $M_{0}(G)$ has a symmetric maximal ideal off the Silov boundary", Proc. London Math. Soc. 27, 484-504.

G. Brown (1975), "Riesz products and generalized characters", Proc. London Math. Soc. 30, 209-238.

G. Brown (1977), "Singular infinitely divisible distributions whose characteristic functions vanish at infinity", Math. Proc. Camb. Phil. Soc. 82, 277-287.

G. Brown (1977), "Fourier transforms on the line and Gronwall's theorem", J. London Math. Soc. (to appear).

G. Brown and W. Moran (1973), "A dichotomy for infinite convolutions of discrete measures", Proc. Camb. Phil. Soc. 73, 307-316.

G. Brown and W. Moran (1974), "On orthogonality of Riesz products", Proc. Camb. Phil. Soc. 76, 173-181.

G. Brown and W. Moran (1975), "Products of random variables and Kakutani's criterion for orthogonality of product measures", J. London Math. Soc. 10, 401-405.

G. Brown and W. Moran (1975), "Coin tossing and powers of singular measures", Math. Proc. Camb. Phil. Soc. 77, 349-364.

T. H. Gronwall (1921), "On the Fourier coefficients of a continuous function", Bull. Amer. Math. Soc. 27, 320-321.

E. Hewitt and G. Ritter (1976), "Úber die Integrierbarkeit von Fourier-Transformierten auf Gruppen; Teil I: Stetige Funktionen mit kompaktem Träger und eine Bemerkung über hyperbolische Differentialoperatoren", Math. Ann. 224, 77-96.

E. Hewitt and G. Ritter (1977), "On the integrability of Fourier transforms on groups; Part II: Fourier-Stieltjes transforms of singular measures", Proc. Roy. Irish Acad. 76A, 265-287.

E. Hewitt and H. S. Zuckerman (1967), "Singular measures with absolutely continuous squares", Proc. Camb. Phil. Soc. 62, 399-420; corrigendum ibid. 63, 367-368.

H. J. Reiter (1968), Classical Harmonic Analysis and Locally Compact Abelian Groups (Oxford University Press, 1968).

S. Saeki (1977), "On infinite convolution products of discrete probability measures", J. London Math. Soc. 16, 172-176.

R. Salem and A. Zygmund (1947), "On a theorem of Banach", Proc. Nat. Acad. Sci. U.S.A. 33, 293-295.

K. Stromberg (1968), "Large families of singular measures having absolutely continuous convolution squares", Proc. Camb. Phil. Soc. 64, 1015-1022.

A. Zygmund (1959), Trigonometric Series, Vols. I and II (Cambridge University Press, 1959).

School of Mathematics

University of New South Wales

P.O. Box 1

Kensington NSW 2033

Australia 